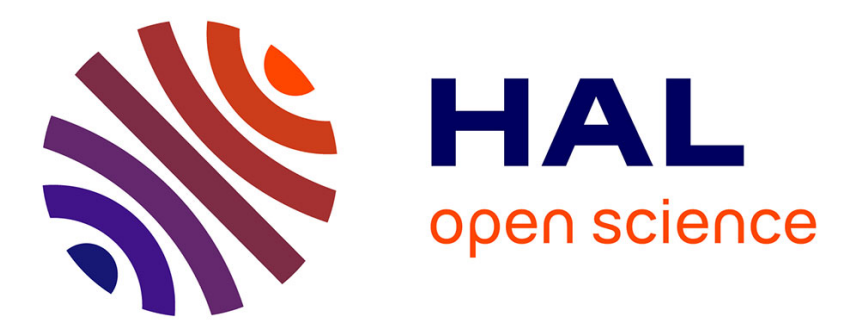

\title{
Analytical solution and Bayesian inference for interference pumping tests in fractal dual-porosity media
}

Mohamed Hayek, Anis Younes, Jabran Zouali, Noura Fajraoui, Marwan Fahs

\section{To cite this version:}

Mohamed Hayek, Anis Younes, Jabran Zouali, Noura Fajraoui, Marwan Fahs. Analytical solution and Bayesian inference for interference pumping tests in fractal dual-porosity media. Computational Geosciences, 2018, 22 (1), pp.413-421. 10.1007/s10596-017-9701-9 . hal-03527143

\section{HAL Id: hal-03527143 \\ https://hal.science/hal-03527143}

Submitted on 15 Jan 2022

HAL is a multi-disciplinary open access archive for the deposit and dissemination of scientific research documents, whether they are published or not. The documents may come from teaching and research institutions in France or abroad, or from public or private research centers.
L'archive ouverte pluridisciplinaire HAL, est destinée au dépôt et à la diffusion de documents scientifiques de niveau recherche, publiés ou non, émanant des établissements d'enseignement et de recherche français ou étrangers, des laboratoires publics ou privés. 
2 Analytical solution and Bayesian inference for interference pumping tests in fractal dual-porosity media

4

5

Mohamed Hayek ${ }^{1}$, Anis Younes ${ }^{\star}, 2,3,4$, Jabran Zouali², Noura Fajraoui ${ }^{5}$, Marwan Fahs $^{2}$

6 ${ }^{2}$ LHyGES, Université de Strasbourg/EOST, CNRS, 1 rue Blessig, 67084 Strasbourg, France ${ }^{3}$ IRD UMR LISAH, F-92761 Montpellier, France ${ }^{4}$ LMHE, Ecole Nationale d'Ingénieurs de Tunis, Tunisie ${ }^{5}$ Chair of Risk, Safety and Uncertainty Quantification, ETH Zurich, Switzerland

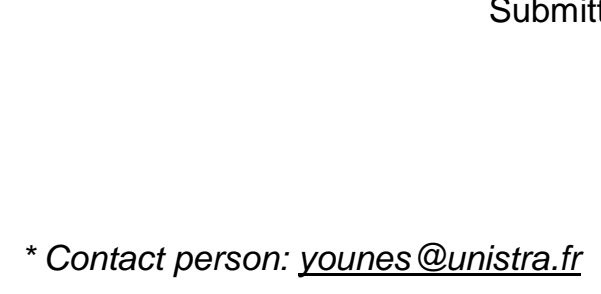




\section{Abstract}

23 A new analytical solution is developed for interference hydraulic pumping tests in fractured

24 porous media using the dual-porosity concept. Heterogeneous fractured reservoirs are consid-

25 ered with hydrodynamic parameters assumed to follow power-law functions in radial dis-

26 tance. The developed analytical solution is verified by comparison against a finite volume

27 numerical solution. The comparison shows that the numerical solution converges toward the

28 analytical one when the size of the time step decreases. The applicability of the fractal dual-

29 porosity model is then assessed by investigating the identifiability of the parameters from a

30 synthetic interference pumping test with a set of noisy data using Bayesian parameter infer-

31 ence. The results show that if the storage coefficient in the matrix is fixed, the rest of the pa-

32 rameters can be appropriately inferred, otherwise, the identification of the parameters is faced

33 with convergence problems because of equifinality issues.

34 Keywords: Fractured porous media, interference pumping test, dual-porosity, fractal media, 35 Bayesian inversion. 


\section{INTRODUCTION}

38 Simulation of interference pumping tests in porous media allows testing different prospective

39 exploitation scenarios at low costs. These tests consist in pumping a well at a controlled flow

40 rate and measuring the water level response (drawdown) in one or more surrounding observa-

41 tion wells. Pumping tests in fractured porous media have been discussed by several authors

42 (see [1-8], among others). Fractured media are often addressed using the dual-porosity model

43 suggested by Barenblatt et al. [9]. The main idea of this model is to consider two overlapping

44 continua: $(i)$ the fractures which carry the major part of water to the pumping well and (ii) the

45 low permeability rock matrix which feeds fluid to the fractures. At each point of the domain,

46 water flow between the fractures and matrix are coupled via a linear exchange term propor-

47 tional to the difference in hydraulic head between the two continua. As stated by De Smedt

48 [8], the simulation of interference pumping tests is often based on numerical models which

49 cannot insure full control of numerical accuracy [10]. Therefore, analytical solutions are high-

50 ly sought-after because $(i)$ they provide valuable insights into fundamental physics, as they

51 are free of numerical errors and (ii), although limited to simplified situations; they allow vali-

52 dation of more complex numerical codes.

53 In the context of interference pumping tests in fractured porous media, an analytical solution

54 has been developed for homogenous aquifers by De Smedt [8]. Furthermore, Delay et al. [7]

55 developed a numerical solution using the dual-medium approach to describe radial convergent

56 flow in heterogeneous fractal media where power laws in space are used for the hydrodynam-

57 ic parameters. As a consequence, parameter values decrease with the distance between the

58 pumped well and the observed ones. This type of scaling laws inherits from theoretical works

59 on fractals [11] and has been used by several authors for single porous media ([12-15], among

60 others). Hence, the main objective of this paper is to develop, for the first time, an analytical

61 solution for the mathematical model describing interference pumping tests in heterogeneous 
62 fractal dual media. Applicability of the model is then assessed by investigating identifiability

63 of the different parameters from a synthetic pumping test with a set of noisy data using Statis-

64 tical Calibration (SC) [16]. The latter means refining the prior distribution of uncertain pa-

65 rameters based on matching simulation outputs with data [17]. In this work, the SC is per-

66 formed with the DREAM(ZS) software [16] based on the Markov Chain Monte Carlo process

67 (MCMC). DREAM(ZS) computes multiple sub-chains in parallel to thoroughly explore the

68 parameter space. Taking the last $25 \%$ of individuals of the MCMC (when the chains have

69 converged) yields multiple sets of parameters that adequately fit the model onto observations.

70 These sets can then be used to estimate the updated parameter distributions and therefore the

71 best estimates of the parameters and the associate confidence intervals.

\section{MATHEMATICAL MODEL}

73 We assume a horizontal, isotropic, confined and infinite aquifer with a uniform thickness. The 74 pumping well only draws water from the fractures, has a constant rate and fully penetrates the aquifer. Using the dual-porosity concept, the flow in fractured porous media can be described by the following equations $[18,19]$ :

$$
S_{f} \frac{\partial h_{f}}{\partial t}=\nabla \cdot\left(K_{f} \nabla h_{f}\right)+\alpha\left(h_{m}-h_{f}\right)
$$

$$
S_{m} \frac{\partial h_{m}}{\partial t}=\alpha\left(h_{f}-h_{m}\right)
$$

79 where indexes $m$ and $f$ refer to the matrix and fracture continua, respectively, $S\left[L^{-1}\right]$ is the

80 specific storage, $h[L]$ is the hydraulic head, $K_{f}\left[L T^{-1}\right]$ is the hydraulic conductivity of the 81 fractures, $\alpha\left[L^{-1} T^{-1}\right]$ is the exchange rate coefficient between the fractures and the matrix. 82 Following Warren and Root [18], equation (2) assumes pseudo-steady flow in the matrix con- 

mulated as:

$$
S_{f}(r) \frac{\partial h_{f}(r, t)}{\partial t}=\frac{1}{r} \frac{\partial}{\partial r}\left[r K_{f}(r) \frac{\partial h_{f}(r, t)}{\partial r}\right]+\alpha(r)\left[h_{m}(r, t)-h_{f}(r, t)\right]
$$

$$
S_{m}(r) \frac{\partial h_{m}(r, t)}{\partial t}=\alpha(r)\left[h_{f}(r, t)-h_{m}(r, t)\right]
$$

where $r[L]$ is the radial coordinate with the center of the pumping well as origin.

The drawdown in an observation well intercepting the fractures can be expressed as follows

$$
\begin{gathered}
S_{f}(r) \frac{\partial H_{f}(r, t)}{\partial t}=\frac{1}{r} \frac{\partial}{\partial r}\left(r K_{f}(r) \frac{\partial H_{f}(r, t)}{\partial r}\right)+\alpha(r)\left[H_{m}(r, t)-H_{f}(r, t)\right] \\
S_{m}(r) \frac{\partial H_{m}(r, t)}{\partial t}=\alpha(r)\left[H_{f}(r, t)-H_{m}(r, t)\right]
\end{gathered}
$$

where $H_{f}=h_{0}-h_{f}$ and $H_{m}=h_{0}-h_{m}$ are respectively the drawdown in the fracture and in the rock matrix. Initially, a uniform hydraulic head $\left(h_{0}\right)$ is assumed in both the fracture and matrix continua. Therefore, the drawdowns satisfy the following initial conditions:

$$
H_{f}(r, 0)=H_{m}(r, 0)=0
$$

Far away from the pumping well, the drawdowns are assumed to be zero

$$
\lim _{r \rightarrow \infty} H_{f}(r, t)=\lim _{r \rightarrow \infty} H_{m}(r, t)=0
$$

97 The limit where the radius approaches zero corresponds to the well withdrawal rate

$$
\lim _{r \rightarrow 0}\left[-2 \pi r B K_{f}(r) \frac{\partial H_{f}(r, t)}{\partial r}\right]=Q_{0}, \quad t>0
$$

where $B$ is the thickness of the aquifer and $Q_{0}$ is the pumping flow rate. 

in the case of many wells with variable rates [20] and by considering transient flow from block to fissure and skin between the fissure and the block [21].

105 In this work, following Delay et al. [7], we consider dual porosity media in which the hydro-

106 dynamic parameters are power-law functions in space (decreasing functions of the radial dis-

107 tance $r$ between the pumping well and the observed one). Moreover, for sake of simplicity, we assume the same functions for the exchange rate coefficient $\alpha$ and for the storage coefficients $S_{f}$ and $S_{m}$. Hence, the values of the hydraulic parameters vary with respect to radial distance according to the following power-law functions:

$$
\begin{array}{rlrl}
K_{f}(r) & =K_{f_{0}} r^{-a} ; & S_{f}(r)=S_{f_{0}} r^{-b} \\
S_{m}(r)=S_{m_{0}} r^{-b} ; & \alpha(r)=\alpha_{0} r^{-b}
\end{array}
$$

112 where the power-law exponents $a$ and $b$ are assumed to be positive.

\section{ANALYTICAL SOLUTION}

\section{$114 \quad 3.1$ Analytical solution in the Laplace domain}

115 In this section, we follow the strategy developed by De Smedt [8] to derive the analytical so-

116 lution of the system formed by equations (5)-(10). The solution is derived using the Laplace

117 transform technique with respect to time variable $t$. The Laplace transform of a time118 dependent function $y$ is

$$
\bar{y}(p)=L[y(t) ; t \rightarrow p]=\int_{0}^{\infty} y(t) e^{-p t} \mathrm{~d} t
$$

$$
p S_{f_{0}} r^{-b} \bar{H}_{f}(r, p)=\frac{K_{f_{0}}}{r} \frac{\partial}{\partial r}\left[r^{1-a} \frac{\partial \bar{H}_{f}(r, p)}{\partial r}\right]+\alpha_{0} r^{-b}\left[\bar{H}_{m}(r, p)-\bar{H}_{f}(r, p)\right]
$$

$$
p S_{m_{0}} \bar{H}_{m}(r, p)=\alpha_{0}\left[\bar{H}_{f}(r, p)-\bar{H}_{m}(r, p)\right]
$$


123 Equation (13) can be rearranged

$$
\bar{H}_{m}(r, p)=\frac{\alpha_{0} / S_{m_{0}}}{p+\alpha_{0} / S_{m_{0}}} \bar{H}_{f}(r, p)
$$

125 Substituting (14) into (12) yields

$$
r^{2} \frac{\partial^{2} \bar{H}_{f}}{\partial r^{2}}+(1-a) r \frac{\partial \bar{H}_{f}}{\partial r}-\frac{S_{f_{0}}}{K_{f_{0}}}\left[p+\frac{\left(\alpha_{0} / S_{f_{0}}\right) p}{p+\alpha_{0} / S_{m_{0}}}\right] r^{a-b+2} \bar{H}_{f}=0
$$

127 Equation (15) is a second order ordinary differential equation; its general solution is of the

128 form (cf. Polyanin and Zaitsev [22], page 159, equation (127))

$$
\bar{H}_{f}(r, p)=r^{\frac{a}{2}}\left\{C_{1} J_{v}\left[\frac{2 i S_{f_{0}}^{1 / 2} r^{n / 2}}{n K_{f_{0}}^{1 / 2}} \sqrt{\mathrm{A}(p)}\right]+C_{2} Y_{v}\left[\frac{2 i S_{f_{0}}^{1 / 2} r^{n / 2}}{n K_{f_{0}}^{1 / 2}} \sqrt{\mathrm{A}(p)}\right]\right\}
$$

130 where $n=a-b+2, v=\frac{a}{n}, \mathrm{~A}(p)=p+\frac{\left(\alpha_{0} / S_{f_{0}}\right) p}{p+\alpha_{0} / S_{m_{0}}}, J_{v}(z)$ and $Y_{v}(z)$ are the Bessel functions

131 of the first and second kinds, respectively. The parameters $C_{1}$ and $C_{2}$ are constant that can be

132 calculated using boundary conditions.

133 In the Laplace domain, the boundary conditions become

$$
\lim _{r \rightarrow \infty} \bar{H}_{f}(r, p)=0
$$

135 and

$$
\lim _{r \rightarrow 0}\left[-2 \pi \mathrm{B} K_{f_{0}} r^{1-a} \frac{\partial \bar{H}_{f}(r, p)}{\partial r}\right]=\frac{Q_{0}}{p}
$$

137 The solution of (15), taking into account the boundary conditions (17) and (18) is

$$
\bar{H}_{f}(r, p)=\frac{2 r^{\frac{a}{2}}}{n^{1-v} \pi \Gamma(1-v) \mathrm{B} K_{f_{0}}^{1-v / 2} S_{f_{0}}^{v / 2}} \frac{\bar{Q}(p) K_{v}\left[\frac{2 S_{f_{0}}^{1 / 2} r^{n / 2}}{n K_{f_{0}}^{1 / 2}} \sqrt{p+\frac{\left(\alpha_{0} / S_{f_{0}}\right) p}{p+\alpha_{0} / S_{m_{0}}}}\right]}{\left[\sqrt{p+\frac{\left(\alpha_{0} / S_{f_{0}}\right) p}{p+\alpha_{0} / S_{m_{0}}}}\right]^{v}}
$$


139 where $K_{v}$ is the modified Bessel function of the second kind of order $v$. More details about

140 the derivation of the solution (19) are given in the Appendix.

\subsection{Analytical solution in the time domain}

143 The solution in the time domain $H_{f}(r, t)$ is sought using the convolution theorem of the La144 place transform [23]

$$
L\left[\int_{0}^{t} y(\tau, t-\tau) \mathrm{d} \tau ; t \rightarrow p\right]=L\left\{L\left[y\left(t_{1}, t_{2}\right) ; t_{2} \rightarrow p\right] ; t_{1} \rightarrow p\right\}
$$

146 Hence, equation (19) is written as

$$
\bar{H}_{f}(r, p)=\bar{H}_{f}\left(r ; p_{1}, p_{2}\right)=\frac{2 Q_{0} r^{\frac{a}{2}}}{n^{1-v} \pi \Gamma(1-v) \mathrm{B} K_{f_{0}}^{1-v / 2} S_{f_{0}}^{v / 2}} \frac{K_{v}\left[\frac{2 S_{f_{0}}^{1 / 2} r^{n / 2}}{n K_{f_{0}}^{1 / 2}} \sqrt{p_{1}+\frac{\left(\alpha_{0} / S_{f_{0}}\right) p_{2}}{p_{2}+\alpha_{0} / S_{m_{0}}}}\right]}{p_{2}\left[\sqrt{p_{1}+\frac{\left(\alpha_{0} / S_{f_{0}}\right) p_{2}}{p_{2}+\alpha_{0} / S_{m_{0}}}}\right]^{v}}
$$

where $p_{1}=p_{2}=p$ but different indexes are used to indicate that the inverse transformation is

150 The different indexes used in (21) indicate that the inverse Laplace transform is developed in pressed as follows

$$
L^{-1}\left[\bar{H}_{f}\left(r ; p_{1}, p_{2}\right) ; p_{1} \rightarrow t_{1}\right]=\frac{n^{v} Q_{0} e^{-\frac{S_{f_{0} r^{n}}}{2 n^{2} K_{f_{0}} t_{1}}} \cdot W_{\frac{1-v}{2}, \frac{v}{2}}\left(\frac{S_{f_{0}} r^{n}}{n^{2} K_{f_{0}} t_{1}}\right)}{\pi \Gamma(1-v) \mathrm{B} K_{f_{0}}^{\frac{1-v}{2}} S_{f_{0}}^{\frac{1+v}{2}} r^{\frac{2-b}{2}}} \cdot \frac{e^{-\frac{\left(\alpha_{0} / S_{f_{0}}\right) t_{1} p_{2}}{p_{2}+\alpha_{0} / S_{m_{0}}}}}{p_{2}}
$$
variable $p_{1}$ and $W_{\kappa, \mu}(z)$ is the Whittaker function (Abramowitz and Stegun [24] p. 505). 
156 In the second step, the inverse Laplace transform with respect to $p_{2}$ is obtained using the in-

157 verse transform of the $J$-function [25] based on the formula $L^{-1}\left[\frac{1}{p} e^{-\frac{\alpha p}{p+\beta}} ; p \rightarrow t\right]=J(\alpha, \beta t)$

158 which yields

159

$$
\bar{H}_{f}\left(r ; p_{1}, p_{2}\right)=\frac{n^{v} Q_{0} e^{-\frac{S_{f_{0}} r^{n}}{2 n^{2} K_{f_{0} t_{1}}}} \cdot t_{1}^{\frac{v-1}{2}} \cdot W_{\frac{1-v}{2}, \frac{v}{2}}\left(\frac{S_{f_{0}} r^{n}}{n^{2} K_{f_{0}} t_{1}}\right) \cdot J\left(\frac{\alpha_{0} t_{1}}{S_{f_{0}}}, \frac{\alpha_{0} t_{2}}{S_{m_{0}}}\right)}{2 \pi \Gamma(1-v) \mathrm{B} K_{f_{0}}^{\frac{1-v}{2}} S_{f_{0}}^{\frac{1+v}{2}} r^{\frac{2-b}{2}}}
$$

160 The Bessel integral $J$-function is defined as follows $[8,26]$

161

$$
J(a, b)=1-e^{-b} \int_{0}^{a} e^{-\lambda} I_{0}(2 \sqrt{b \lambda}) \mathrm{d} \lambda=e^{-a-b} \sum_{n=0}^{\infty} \frac{b^{n}}{n !} \sum_{m=0}^{n} \frac{a^{m}}{m !}
$$

162 In view of (20) and (23), the analytical expression of $H_{f}(r, t)$ is

$$
H_{f}(r, t)=\frac{n^{v} Q_{0}}{2 \pi \Gamma(1-v) \mathrm{B} K_{f_{0}}^{\frac{1-v}{2}} S_{f_{0}}^{\frac{1+v}{2}}} r^{\frac{2-b}{2}} \int_{0}^{t} \tau^{\frac{v-1}{2}} \cdot e^{-\frac{S_{f_{0}} r^{n}}{2 n^{2} K_{f_{0}} \tau}} \cdot W_{\frac{1-v}{2}, \frac{v}{2}}\left(\frac{S_{f_{0}} r^{n}}{n^{2} K_{f_{0}} \tau}\right) \cdot J\left(\frac{\alpha_{0} \tau}{S_{f_{0}}}, \frac{\alpha_{0}(t-\tau)}{S_{m_{0}}}\right) \mathrm{d} \tau
$$

167 In the case of homogeneous media $(a=b=0)$, we have $W_{\frac{1}{2}, 0}(z)=z^{\frac{1}{2}} e^{-\frac{z}{2}}$ and (25) reduces to the De Smedt [8] solution:

$$
H_{f}(r, t)=\frac{Q_{0}}{4 \pi \mathrm{B} K_{f_{0}}} \int_{0}^{t} \tau^{-1} e^{-\frac{S_{f_{0}} r^{2}}{4 K_{f_{0}} \tau}} \cdot J\left(\frac{\alpha_{0} \tau}{S_{f_{0}}}, \frac{\alpha_{0}(t-\tau)}{S_{m_{0}}}\right) \mathrm{d} \tau
$$




\section{COMPARISON TO NUMERICAL SOLUTIONS}

174 We numerically solve two interference pumping tests dealing with homogeneous and fractal

175 dual media. For each test, the numerical solution is compared against the corresponding ana-

176 lytical solution using (25). The numerical solution of the system (5)-(10) is developed using

177 the standard Finite Volume (FV) method. The domain of size $L=10,000 \mathrm{~m}$ is discretized us-

178 ing a uniform spatial discretization of size $\Delta r=5 \mathrm{~m}$. A similar solution is obtained using a

179 finer spatial discretization of size $\Delta r=1 \mathrm{~m}$ which shows the weak sensitivity of the numerical

180 solution to the spatial discretization. The duration of the simulation is $2 \times 10^{6} \mathrm{~s}$. Three time

181 step sizes are investigated with $\Delta t=10^{5} \mathrm{~s}, \Delta t=5 \times 10^{4} \mathrm{~s}$ and $\Delta t=10^{4} \mathrm{~s}$. The pumping flow rate

182 is $Q_{0}=5 \mathrm{~m}^{3} / \mathrm{h}$. The initial head in the aquifer is $h_{0}=100 \mathrm{~m}$ and the aquifer thickness is

$183 B=100 \mathrm{~m}$. The specific storage of the fractures and rock matrix are respectively $S_{f_{0}}=5 \times 10^{-6}$

$184 \mathrm{~m}^{-1}$ and $S_{m_{0}}=10^{-4} \mathrm{~m}^{-1}$. The hydraulic conductivity of the fractures is $K_{f_{0}}=10^{-4} \mathrm{~m} / \mathrm{s}$ and the

185 rate of exchange between the matrix and the fractures is $\alpha_{0}=5 \times 10^{-11} \mathrm{~m}^{-1} \mathrm{~s}^{-1}$.

186 Figure 1 depicts the drawdown in both the fracture and matrix at $10 \mathrm{~m}$ and $100 \mathrm{~m}$. The draw-

187 down in the fissure starts at early times because storage of the fissures is immediately effec-

188 tive at the start of the pumping test, while storage of the matrix needs much more time to be-

189 come noticeable. A flattening of the drawdown curve in the fissure can be observed at inter-

190 mediate pumping time which is typical for double-porosity behavior because of the transition

191 from fracture to matrix water storage release.

192 The numerical FV solution is compared to the analytical solution (25) in the case of homoge-

193 neous $(a=b=0)$ and fractal $(a=0.6$ and $b=0.4)$ dual media. The comparison is performed

194 at two observation wells located respectively at distances $r_{1}=10 \mathrm{~m}$ and $r_{2}=100 \mathrm{~m}$ from the

195 pumping well. Significant discrepancies can be observed between analytical and numerical 
196 solutions when the latter is evaluated using a large time step size (Figure 2). The drawdown in

197 the case of fractal dual media is larger than in homogeneous dual media because of the reduc-

198 tion of the hydrodynamic parameters with the distance $r$ from the pumping well. In fact, in

199 the case of fractal dual media, the permeability of the fractures is proportional to $r^{-0.6}$ where-

200 as the exchange rate and the specific storage of both the fractures and the matrix are propor-

201 tional to $r^{-0.4}$. Thereby, the discrepancy between analytical and numerical solutions (Figure

202 2) is more pronounced with dual fractal media than with homogeneous dual media. For both

203 homogeneous and fractal dual media, the numerical solution converges toward the analytical

204 solution (25) when the size of the time step decreases (Figure 2). Note that the reduction of

205 the permeability of the fractures with respect to the distance $r$ is also responsible, via the

206 Whittaker function in equation (25), of the delay for the stabilisation of the solution in the

207 fractal case (Figure $2 \mathrm{~b}$ ) compared to the homogeneous case (Figure 2a).

2085 BAYESIAN INVERSION OF A PUMPING TEST IN FRACTAL DUAL MEDIA

209 In practical applications, most of the hydraulic parameters cannot be measured directly and

210 should be estimated using an inversion procedure. In the case of fractal dual media, the inver-

211 sion can be challenging because of model equifinality, implying that different parameter com-

212 binations can fit the model responses to data. To assess the applicability of the fractal dual

213 porosity model, the identifiability of the parameters of the analytical solution (25) is assessed

214 using a synthetic interference pumping test with a set of noisy data. Parameter identification is

215 performed in a Bayesian framework where the prior knowledge about the parameters and the

216 observed data are merged to define the joint posterior probability distribution function (pdf) of

217 the parameters. In this work, the pdf is performed using the $\operatorname{DREAM}_{(\mathrm{ZS})}$ software [16] based

218 on the Markov Chain Monte Carlo (MCMC) sampler. DREAM(ZS) generates random se-

219 quences of parameter sets that asymptotically converge toward the target joint posterior dis- 
exploring the entire parameter space of the posterior distribution of the parameters and pro-

vides the pairwise parameter correlations and the uncertainty of model predictions.

223 The Bayes theorem states that the probability density function of the model parameters conditioned onto data can be expressed as:

$$
p\left(\boldsymbol{\xi} \mid \boldsymbol{y}_{\text {mes }}\right) \propto p\left(\boldsymbol{y}_{\text {mes }} \mid \boldsymbol{\xi}\right) p(\boldsymbol{\xi}),
$$

where $p\left(\boldsymbol{\xi} \mid \boldsymbol{y}_{\text {mes }}\right)$ is the likelihood function measuring how well the model fits the observations $\boldsymbol{y}_{\text {mes }}$, and $p(\xi)$ is the prior information about the parameter before the observations are made. In this work, a Gaussian distribution defines the likelihood function because the observations are simulated and corrupted with Gaussian errors. Hence, the parameter posterior distribution is expressed as:

$$
p\left(\boldsymbol{\xi} \mid \boldsymbol{y}_{\text {mes }}\right) \propto \sigma_{h}^{-N_{h}} \exp \left(-\frac{S S_{h}(\boldsymbol{\xi})}{2 \sigma_{h}^{2}}\right)
$$

where $S S_{h}(\xi)$, are the sums of the squared differences between the observed and modeled

233 drawdowns. For instance, $S S_{h}(\xi)=\sum_{k=1}^{N h}\left(h_{\text {mes }}^{(k)}-h_{\text {mod }}^{(k)}(\xi)\right)^{2}$, which includes the observed $h_{\text {mes }}^{(k)}$

234 and predicted $h_{\text {mod }}^{(k)}$ drawdowns at time $t_{k}$ for the number of observations $N_{h}$.

235 A synthetic pumping test is generated by running the analytical solution using the reference

236 parameter values given in Table 1 for $K_{f_{0}}, S_{f_{0}}, \alpha_{0}, a$ and $b$. As in Fahs et al. [28], the stor-

237 age coefficient in the matrix $S_{m_{0}}$ is fixed in this study to be $10^{-4} \mathrm{~m}^{-1}$.

238 Delay et al [7] showed that results of calibration improved when several drawdown curves at 239 several locations are inverted at once. In the sequel, the analytical solution is run for a long 240 period of $2 \times 10^{7} \mathrm{~s}$ to calculate the drawdown at four observation wells located respectively at 241 $r_{1}=1 \mathrm{~m}, r_{2}=10 \mathrm{~m}, r_{3}=100 \mathrm{~m}$ and $r_{4}=500 \mathrm{~m}$ from the pumping well. Four datasets of 2000 
242 values each (corresponding to the drawdown at the observation wells measured each $10^{4} \mathrm{~s}$ )

243 are used as target responses for model inversion.

244 Gaussian noise with a standard deviation of $0.01 \mathrm{~m}$ and a mean of zero was added to the target

245 responses. We assume that all hydraulic parameters have uniform prior distributions over the

246 ranges given in Table 1. As recommended in Vrugt et al. [29], we consider that the posterior

247 distribution is stationary if the Gelman and Ruban [30] criterion is less than 1.2 and that the

248 chains are not autocorrelated.

249 The MCMC method was terminated after 15000 model runs. The convergence was reached at

250 around 10000 model runs. Figure 3 shows the results of the identification based on the last

$25125 \%$ of individuals of the MCMC sampler (when the chains have converged). The "on-

252 diagonal" plots in these figures display the inferred parameter distributions, whereas the "off-

253 diagonal" plots represent the pairwise correlations in the MCMC sample. If the drawings are

254 independent, non-sloping scatterplots should be observed. However, if a good value of a giv-

255 en parameter is conditioned by the value of another parameter, then their pairwise scatterplot

256 should show a narrow sloping stripe. The results of Figure 3 show that the five parameters

$257 K_{f_{0}}, S_{f_{0}}, \alpha_{0}, a$ and $b$ are appropriately estimated; they have almost symmetric bell-shaped

258 posterior distributions and have strongly narrowed their prior intervals. The scatter plots in

259 this figure show a moderate correlation between the parameters $a$ and $b$ (a correlation factor

260 of 0.61 ), a high correlation between the parameters $S_{f_{0}}$ and $b$ (a correlation factor of 0.79)

261 and a very high correlation between the parameters $K_{f_{0}}$ and $a$ (a correlation factor of 0.98).

262 The mean and $95 \%$ confidence intervals of the samples that adequately fit the model onto

263 observations are reported in Table 2. From this table, we can see that the parameters $K_{f_{0}}, a$

264 and $b$ are well identified with respectively a mean value of $0.99 \times 10^{-5} \mathrm{~ms}^{-1}, 0.299$ and 0.2 .

265 These values are almost identical to the reference values of Table 1 . The uncertainty on these 
266 parameters is small since the $95 \%$ confidence intervals are respectively $[0.9-1.1] \times 10^{-5} \mathrm{~ms}^{-1}$,

$267[0.28-0.32]$ and [0.17-0.23]. The parameters $S_{f_{0}}$ and $\alpha_{0}$ are less well identified. Indeed, alt268 hough their mean values (respectively $5.03 \times 10^{-5} \mathrm{~m}^{-1}$ and $3.06 \times 10^{-11} \mathrm{~m}^{-1} \mathrm{~s}^{-1}$ ) are very close to 269 the reference values, their posterior uncertainty remains quite large since their confidence 270 intervals are respectively $[3.2-7.6] \times 10^{-5} \mathrm{~m}^{-1}$ and $[2.1-4.5] \times 10^{-11} \mathrm{~m}^{-1} \mathrm{~s}^{-1}$.

271 Finally note that the identification procedure does not converge if the storage coefficient in 272 the matrix $S_{m_{0}}$ is involved in the inversion procedure (not fixed). In that case, the identifica273 tion of the six parameters $K_{f_{0}}, S_{f_{0}}, S_{m_{0}}, \alpha_{0}, a$ and $b$ was not possible. Indeed, in this case, 274 the MCMC algorithm was faced to convergence problem because of equifinality issues.

\section{CONCLUSION}

277 A new analytical solution for interference hydraulic pumping tests in fractal fractured porous 278 media is developed using the dual-porosity concept and power laws in space for all hydrody279 namic parameters. The developed analytical solution is compared to a numerical one obtained 280 with the FV method using different time step sizes. Discrepancies are observed between ana281 lytical and numerical solutions when the latter is used with large time steps. These discrepan282 cies are more pronounced with dual fractal media than with homogeneous dual media. When 283 the size of the time step decreases, the numerical solution converges toward the analytical one 284 for both homogeneous and fractal dual media.

285 The applicability of the fractal dual-porosity model was then assessed by investigating identi286 fiability of the hydraulic parameters from a synthetic interference pumping test with a set of 287 noisy data using the MCMC sampler. The results show that if the storage coefficient in the 
288 matrix is fixed, the rest of the parameters can be appropriately identified, whereas conver289 gence problems can be encountered if all the hydraulic parameters are to be estimated.

290

291 Acknowledgements

292 This work was partially supported by the GdR MoMaS (PACEN/CNRS, ANDRA, BRGM,

293 CEA, EDF, IRSN) France and by the French National Research Agency (ANR) through the 294 program AAP Blanc - SIMI 6 project RESAIN (n॰ANR-12-BS06-0010-02). 
297 Appendix: Derivation of the Analytical Solution in the Laplace Domain

298

299 Using the boundary condition at infinity (17) and the following asymptotic expansions for

300 large arguments $(|z| \rightarrow \infty)$ of $J_{v}$ and $Y_{v}$ [22], $J_{v}(z)=\sqrt{2 / \pi z}\{P(v, z) \cos \chi-Q(v, z) \sin \chi\}$

301 and $Y_{v}(z)=\sqrt{2 / \pi z}\{P(v, z) \cos \chi+Q(v, z) \sin \chi\}$ for $|\arg z|<\pi$ with $\chi=z-\left(\frac{1}{2} v+\frac{1}{4}\right) \pi$,

$302 P(v, z)=\sum_{k=0}^{\infty}(-1)^{k} \frac{(v, 2 k)}{(2 z)^{2 k}} \quad$ and $\quad Q(v, z)=\sum_{k=0}^{\infty}(-1)^{k} \frac{(v, 2 k+1)}{(2 z)^{2 k+1}}, \quad$ equation (16) gives

$303 C_{1}+i C_{2}=0$. Therefore, this latter can be written as

304

$$
\bar{H}_{f}(r, p)=C_{1} r^{\frac{a}{2}} H_{v}^{(1)}\left[\frac{2 i S_{f_{0}}^{1 / 2} r^{n / 2}}{n K_{f_{0}}^{1 / 2}} \sqrt{\mathrm{A}(p)}\right]
$$

305 where $H_{v}^{(1)}$ is the Hankel function of the first kind defined by $H_{v}^{(1)}(z)=J_{v}(z)+i Y_{v}(z)$ for 306 any complex number $z$.

307 The boundary condition at the well (18) is then used to find $C_{1}$. Note that for $v>0$ the 308 Hankel function $H_{v}^{(1)} \sim-\frac{i}{\pi} \Gamma(v)\left(\frac{1}{2} z\right)^{-v}$ as $z$ approaches zero [22], where $\Gamma$ is the Gamma 309 function. Using this expansion we can write

$$
r^{1-a} \frac{\partial H_{f}(r, t)}{\partial r} \sim \frac{i^{1-v} S_{f_{0}}^{v / 2} C_{1}}{2 \pi K_{f_{0}}^{v / 2}}\left\{-r^{-a} n^{v}[\sqrt{A(p)}]^{-v}\{a \Gamma(v)-n \Gamma(1+v)\}+n^{1-v}[\sqrt{A(p)}]^{v} \Gamma(1-v)\right\}
$$


312 as $r$ appraches zero. Using the property of the Gamma function $\Gamma(1+v)=v \Gamma(v)$ for $v>0$,

313 the first term of right hand side of equation (A.2) vanishes since $n v=a$. Substituting (A.2)

314 into (18) we get $C_{1}=-\frac{\bar{Q}(p)}{i^{1-v} n^{1-v} \Gamma(1-v) \mathrm{B} K_{f_{0}}^{1-v / 2} S_{f_{0}}^{v / 2}[\sqrt{A(p)}]^{v}}$.

315 Finally, inserting the value of $C_{1}$ into (A.1) and using the relationship $316 K_{v}(z)=\frac{1}{2} \pi i e^{\frac{1}{2} v \pi i} H_{v}^{(1)}\left(z e^{\frac{1}{2} \pi i}\right)$ for $-\pi<\arg z \leq \frac{1}{2} \pi$, where $K_{v}$ is the modified Bessel func317 tion of the second kind of order $v$, we get the solution in the Laplace domain as defined by 318 (19).

319 


\section{REFERENCES}

322 [1] Hsieh, P.A.: A brief survey of hydraulic tests in fractured rocks. In Faybishenko B, Witherspoon PA, Benson SM (eds.) Dynamics of fluids in fractured rocks, American Geophysical Union Geophysical Monograph 122:59-66 (2000).

[2] Kruseman, G.P., de Ridder, N.A.: Analysis and evaluation of pumping test data. International Institute for Land Reclamation and Improvement Publication, Wageningen (1991).

[3] Batu, V.: Aquifer Hydraulics: a comprehensive guide to hydrogeologic data analysis. John Wiley \& Sons Inc. New York (1998).

[4] Cheng, A.H.D.: Multilayered aquifer systems fundamentals and applications. Marcel

[5] Walton, W.C.: Aquifer test modelling. CRC press, Taylor \& Francis Group, Boca Raton (2006).

[6] Nielsen, K.A.: Fractured aquifers formation evaluation by Well Testing. Trafford Publishing, Canada (2007).

[7] Delay, F., Kaczmaryk, A., Ackerer, P.: Inversion of interference hydraulic pumping tests in both homogeneous and fractal dual media. Adv. in Water Resour. 30(3):314-

[8] De Smedt, F.: Analytical solution Analytical Solution for Constant-Rate Pumping Test in Fissured Porous Media with Double-Porosity Behaviour. Transp Porous Med. 88:479-489 (2011). doi:10.1007/s11242-011-9750-9.

[9] Barenblatt ,G.E., Zheltov, I.P., Kochina, I.N.: Basis concepts in the theory of seepage of homogeneous liquids in fissured rocks. J. Appl. Math. Mech. Engl. Transl. 24(5):12861303 (1960). doi:10.1016/0021-8928(60)90107-6. 
[10] Zhan, H., Wen, Z., Gao, G.: An analytical solution of two-dimensional reactive solute transport in an aquifer aquitard system. Water Resour. Res. 45, W10501. (2009). doi:10.1029/2008WR007479

[11] O'Shaughnessy, B., Procaccia, I.: Diffusion in fractals. Phys Rev A. ;32:3073 (1985) doi:10.1103/PhysRevA.32.3073

[12] Acuna, J.A., Yortsos, Y.C.: Application of fractal geometry to the study of networks of fractures and their pressure transient. Water Resour Res; 31(3):527-40 (1995). doi: 10.1029/94WR02260

[13] Chang, J., Yortsos, Y.C.: Pressure-transient analysis of fractal reservoirs. Soc Petrol Eng J 18710:631-43 (1990) doi:10.2118/18170-PA.

[14] Delay, F., Porel, G., Bernard, S.: Analytical 2D model to invert hydraulic pumping tests in fractured rocks with fractal behavior. Geophys Res Lett. 31(16) (2004). doi:10.1029/2004GL020500.

[15] Le Borgne, T., Bour, O., de Dreuzy, J.R., Davy, P., Touchard, F.: Equivalent mean flow models for fractured aquifers: insights for a pumping test scaling interpretation. Water Resour Res;40(3) (2004). doi:10.1029/2003WR002436.

[16] Laloy, E., Vrugt, J.A.: High-dimensional posterior exploration of hydrologic models using multiple-try DREAM(ZS) and high-performance computing, Water Resour. Res., 48 W01526. (2012). doi:10.1029/2011WR010608.

[17] Campbell, K.: Statistical calibration of computer simulations, Reliability Engineering and System Safety 91 1358-1363 (2006). doi:10.1016/j.ress.2005.11.032

[18] Warren, J.E., Root, P.J.: The behavior of naturally fractured reservoirs. Soc Petrol Eng 3:245-55. (1963). doi:10.2118/426-PA.

[19] Moench, A.F.: Double-porosity models for a fissured groundwater reservoir with fracture skin. Water Resour. Res. 20(7):831-846. (1984). doi:10.1029/WR020i007p00831.

[20] Chang, J., Yortsos, Y. C. A Note On Pressure-Transient Analysis Of Fractal Reservoirs. Society of Petroleum Engineers. (1993). doi:10.2118/25296-PA 
[21] Hamm, S.Y., Bidaux, P. Dual-porosity fractal models for transient flow analysis in fissured rocks: Water Resources Research, v. 32, no. 9, p. 2733-2745 (1996).

374

375

376

377

378

379

380

381

382

383

384

385

386

387

388

389

390

391

392

393

394

395

396

[22] Polyanin, A.D., Zaitsev, V.F. Handbook of Exact Solutions for Ordinary Differential Equations, 2nd ed., 787 pp., Chapman and Hall/ CRC, Boca Raton, Fla (2003).

[23] Sneddon, I.H.: The Use of Integral Transforms, McGraw-Hill, New York (1972).

[24] Abramowitz, M., Stegun, I.A. Handbook of Mathematical Functions With Formulas, Graphs, and Mathematical Tables, Government Printing Off., Washington, D. C. (1972).

[25] Goldstein, F.R.S.: On the mathematics of exchange processes in fixed columns I: Mathematical solutions and asymptotic expansions. Proc. R. Soc. London 219: 151-185. (1953).

[26] De Smedt, F., Wierenga, P.J.: A generalized solution for solute flow in soils with mobile and immobile water. Water Resour. Res. 15(5): 1137-1141. (1979). doi: 10.1029/WR015i005p01137.

[27] Gelman, A., Carlin, J.B., Stren, H.S., Rubin, D.B. Bayesian data analysis (1997), Chapmann and Hall, London.

[28] Fahs, H., Hayek, M., Fahs, M., Younes, A.: An efficient numerical model for hydrodynamic parameterization in 2D fractured dual-porosity media. Adv. Water Resour. 63: 179-193. (2013). doi: 10.1016/j.advwatres.2013.11.008

[29] Vrugt, J.A., Gupta, H.V., Bouten, W., Sorooshian, S. A shuffled complex evolution Metropolis algorithm for optimization and uncertainty assessment for hydrologic model parameters. Water Resour. Res. 39(8):1201 (2003), doi:10.1029/2002WR001642.

[30] Gelman, A., Rubin, D.B. Inference from iterative simulation using multiple sequences. Stat. Sci. 7:457-472 (1992). doi: 10.1214/ss/1177011136 
$399 \quad$ List of Tables

400 Table 1: Reference values, lower and upper bounds of the parameters for a synthetic pumping 401 test case in fractal dual media.

402 Table 2: Mean values and $95 \%$ confidence intervals for the parameters estimated with the 403 MCMC sampler.

404

405

$406 \quad$ List of Figures

407 Figure 1: Drawdown versus time curves for a pumping test in homogeneous and fractal dual 408 media.

409 Figure 2: Comparison between analytical and numerical solutions in the case of $(i)$ homoge410 neous and (ii) fractal dual media.

411 Figure 3: MCMC solutions of the interference pumping test in fractal dual media. The diago412 nal plots represent the inferred posterior probability distribution of model parameters. The off413 diagonal scatterplots represent the value of a parameter $\alpha$ versus that of a parameter $\beta$ in the 414 best solutions inferred by MCMC. 


\section{Table 1}

423

424

\begin{tabular}{|c|c|c|c|}
\hline Parameters & Lower bounds & Upper bounds & Reference values \\
\hline$K_{f_{0}}\left[\mathrm{~ms}^{-1}\right]$ & $5 \times 10^{-7}$ & $5 \times 10^{-4}$ & $1 \times 10^{-5}$ \\
\hline$S_{f_{0}}\left[\mathrm{~m}^{-1}\right]$ & $10^{-6}$ & $10^{-3}$ & $5 \times 10^{-5}$ \\
\hline$\alpha_{0}\left[\mathrm{~m}^{-1} \mathrm{~s}^{-1}\right]$ & $10^{-12}$ & $10^{-9}$ & $3 \times 10^{-11}$ \\
\hline$a[-]$ & 0.1 & 0.5 & 0.3 \\
\hline$b[-]$ & 0.1 & 0.5 & 0.2 \\
\hline
\end{tabular}

425 
428

429

Table 2

430

431

\begin{tabular}{|c|c|c|}
\hline Parameters & Mean & 95\% Conf. Int. \\
\hline$K_{f_{0}}\left[\mathrm{~ms}^{-1}\right]$ & $0.99 \times 10^{-5}$ & {$[0.9-1.1] \times 10^{-5}$} \\
\hline$S_{f_{0}}\left[\mathrm{~m}^{-1}\right]$ & $5.03 \times 10^{-5}$ & {$[3.2-7.6] \times 10^{-5}$} \\
\hline$\alpha_{0}\left[\mathrm{~m}^{-1} \mathrm{~s}^{-1}\right]$ & $3.06 \times 10^{-11}$ & {$[2.1-4.5] \times 10^{-11}$} \\
\hline$a[-]$ & 0.299 & {$[0.28-0.32]$} \\
\hline$b[-]$ & 0.2 & {$[0.17-0.23]$} \\
\hline
\end{tabular}

432

433

434

435

436

437

438

439

440

441 
443

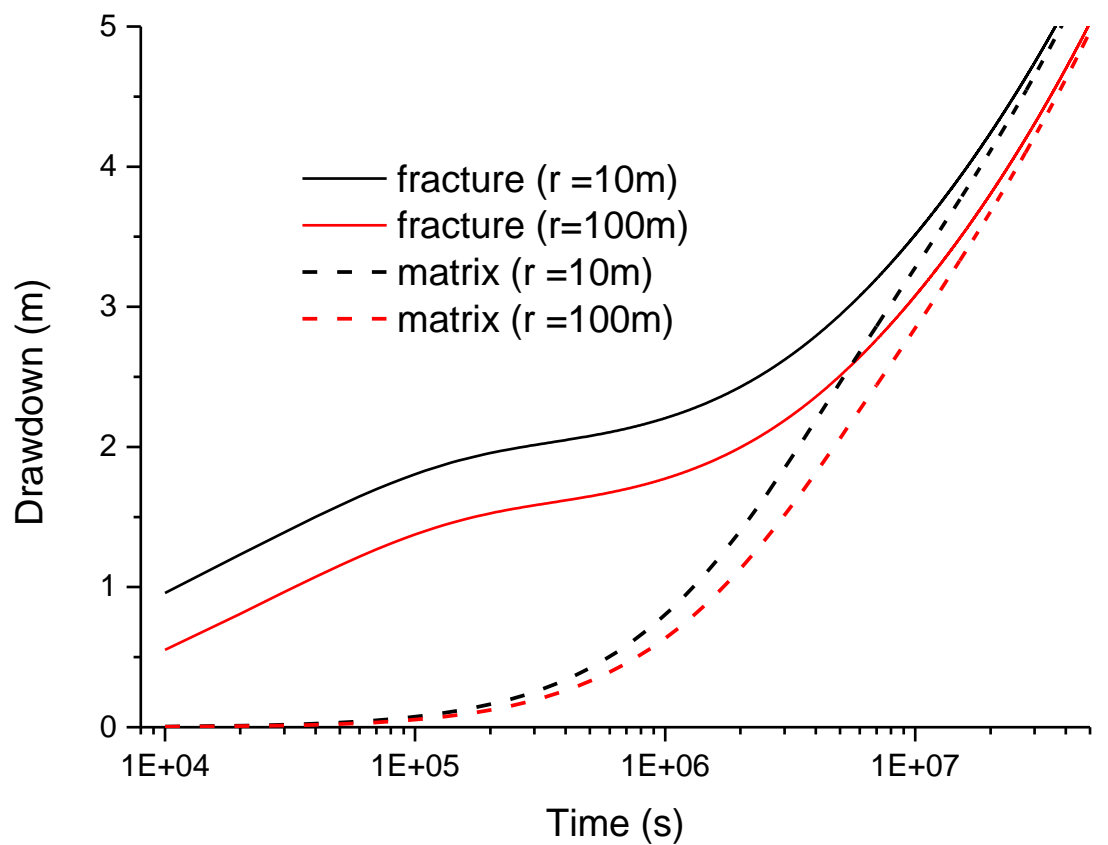

444

445 
$447 \quad$ Figure 2

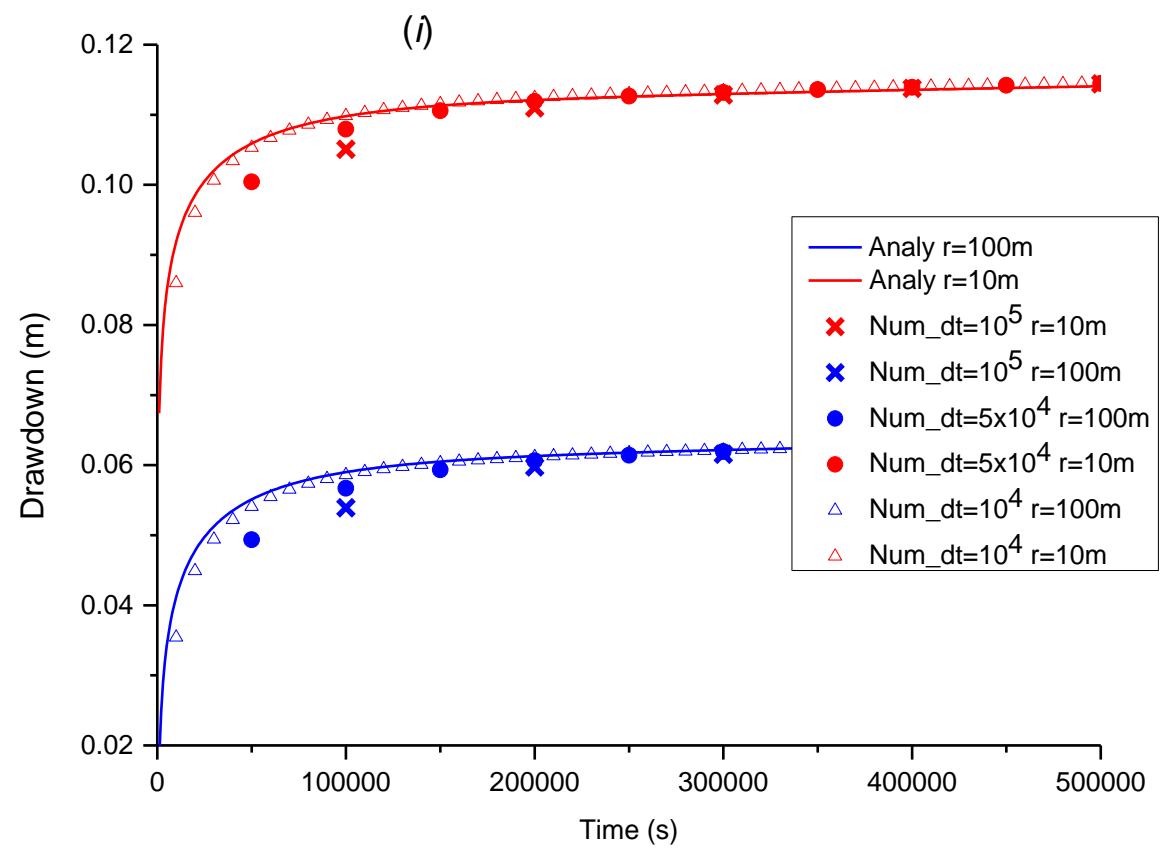

448

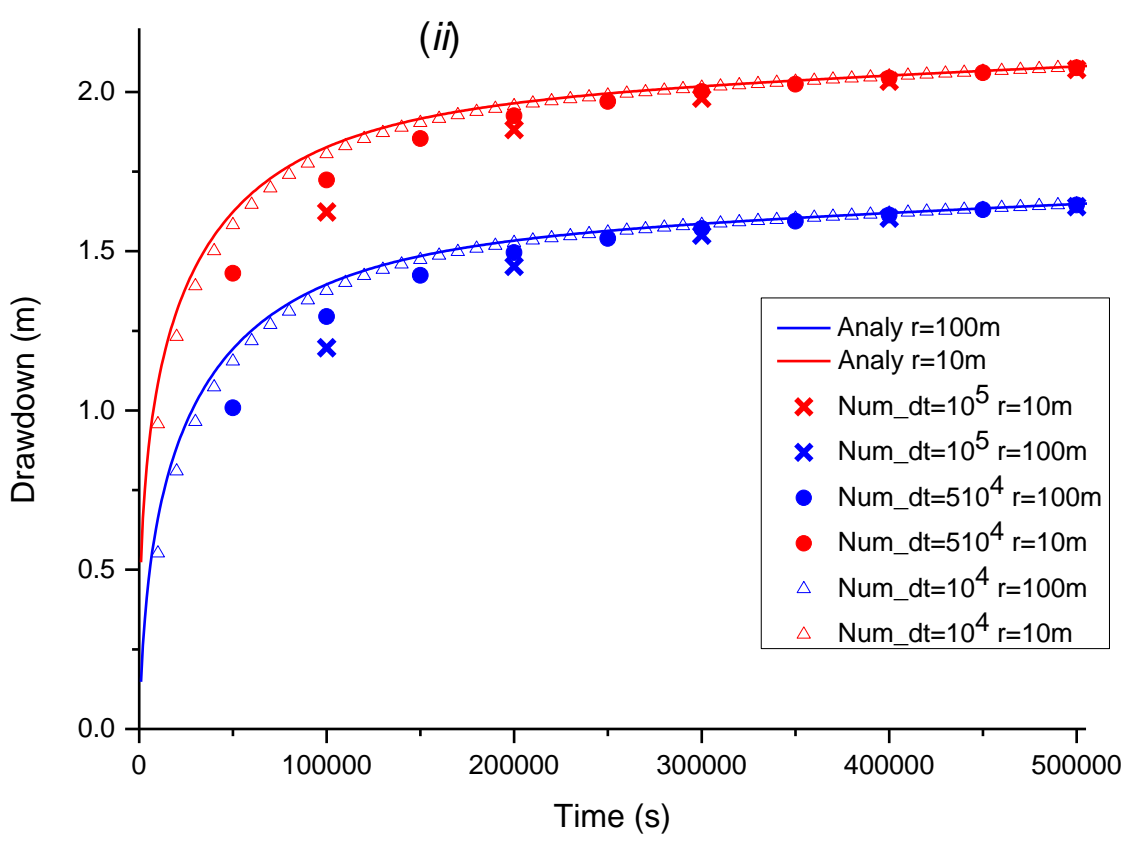


Figure 3

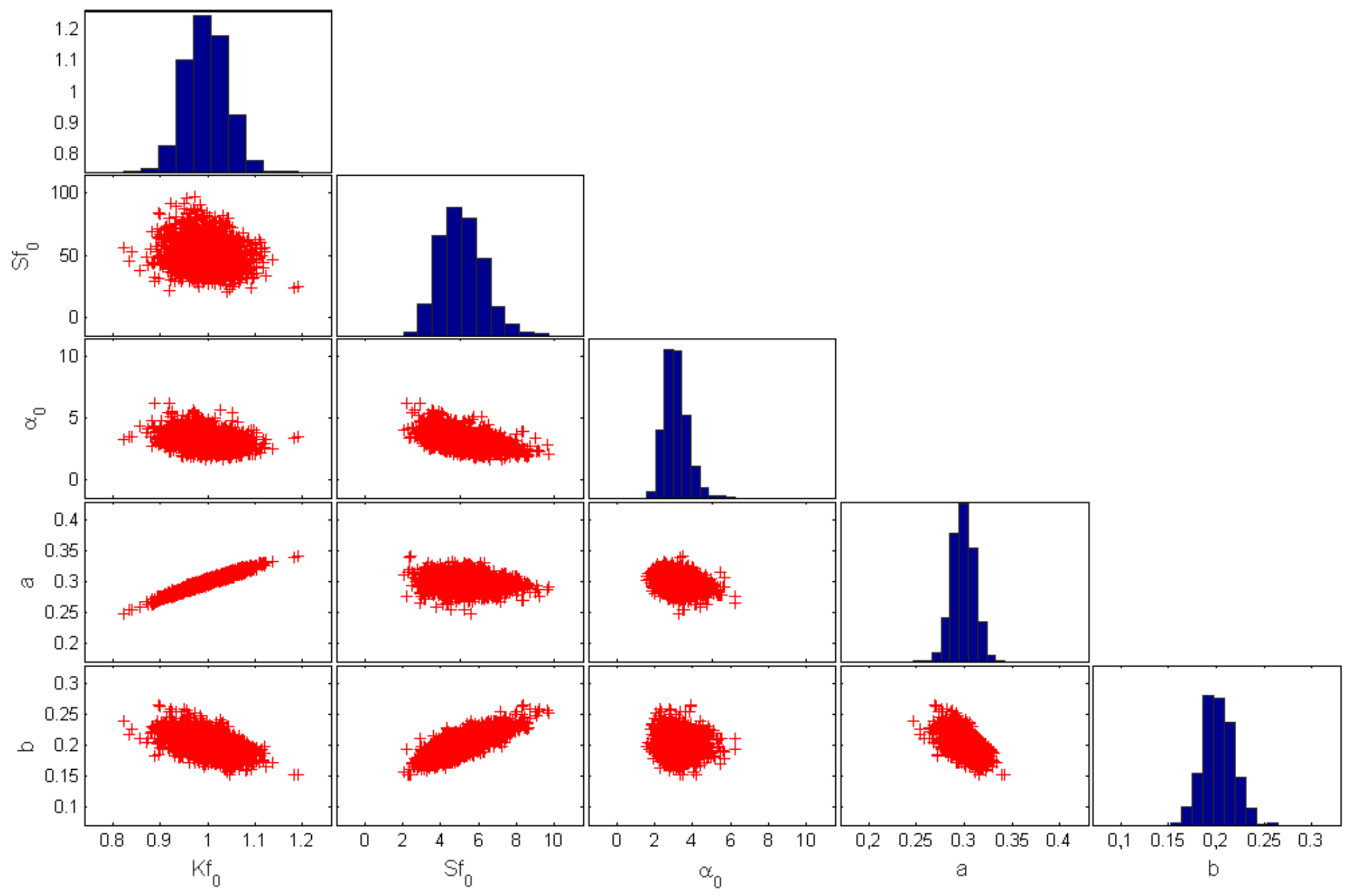

